Published in final edited form as:

Gynecol Oncol. 2013 September ; 130(3): 518-524. doi:10.1016/j.ygyno.2013.06.003.

\title{
A Phase I Clinical Trial of Ad5/3- $\Delta 24$, a Novel Serotype-Chimeric, Infectivity-Enhanced, Conditionally-Replicative Adenovirus (CRAd), in Patients with Recurrent Ovarian Cancer
}

\author{
Kenneth H. Kim ${ }^{1}$, Igor P. Dmitriev², Souheil Saddekni ${ }^{3}$, Elena A. Kashentseva ${ }^{2}$, Raymond \\ D. Harris ${ }^{4}$, Rosemarie Aurigemma ${ }^{5}$, Sejong Bae ${ }^{6}$, Karan P. Singh ${ }^{6}$, Gene P. Siegal ${ }^{7}$, David \\ T. Curiel ${ }^{2}$, and Ronald D. Alvarez ${ }^{8}$ \\ ${ }^{1}$ The Division of Gynecologic Oncology, The University of North Carolina \\ ${ }^{2}$ The Division of Cancer Biology, Washington University School of Medicine \\ ${ }^{3}$ The Department of Radiology, The University of Alabama at Birmingham \\ ${ }^{4}$ Office of Biodefense Research Affairs, DMID, NIAID, National Institutes of Health \\ ${ }^{5}$ Biological Resources Branch, National Cancer Institute \\ ${ }^{6}$ The Biostatistics and Informatics Shared Facility (BBSF), The University of Alabama at \\ Birmingham \\ ${ }^{7}$ The Department of Pathology, The University of Alabama at Birmingham \\ ${ }^{8}$ The Division of Gynecologic Oncology, The University of Alabama at Birmingham
}

\begin{abstract}
Objective-The conditionally replicative adenovirus $\mathrm{Ad} 5 / 3-\Delta 24$ has a type- 3 knob incorporated into the type-5 fiber that facilitates enhanced ovarian cancer infectivity. Preclinical studies have shown that Ad5/3- $\Delta 24$ achieves significant oncolysis and antitumor activity in ovarian cancer models. The purpose of this study was to evaluate in a Phase I trial the feasibility and safety of intraperitoneal (IP) Ad5/3- $\Delta 24$ in recurrent ovarian cancer patients.
\end{abstract}

\begin{abstract}
Methods-Eligible patients were treated with IP Ad5/3- $\Delta 24$ for 3 consecutive days in one of three dose cohorts ranging $1 \times 10^{10}-1 \times 10^{12} \mathrm{vp}$. Toxicity was assessed utilizing CTC grading and efficacy with RECIST. Ascites, serum, and other samples were obtained to evaluate gene transfer, generation of wildtype virus, viral shedding, and antibody response.
\end{abstract}

\begin{abstract}
Results-Nine of 10 patients completed treatment per protocol. A total of 15 vector-related adverse events were experienced in 5 patients. These events included fever or chills, nausea, fatigue, and myalgia. All were grade 1-2 in nature, transient, and medically managed. Of the 8 treated patients evaluable for response, six patients had stable disease and 2 patients had progressive disease. Three patients had decreased CA-125 from pretreatment levels one month
\end{abstract}

(C) 2013 Elsevier Inc. All rights reserved.

Corresponding Author: Ronald D. Alvarez, M.D., University of Alabama at Birmingham, Division of Gynecologic Oncology, 176F Rm 10250, 619 19th Street South, Birmingham, Alabama 35249, rdalvarez@uab.edu, phone: (205) 934-4986, fax: (205) 975-6174.

Publisher's Disclaimer: This is a PDF file of an unedited manuscript that has been accepted for publication. As a service to our customers we are providing this early version of the manuscript. The manuscript will undergo copyediting, typesetting, and review of the resulting proof before it is published in its final citable form. Please note that during the production process errors may be discovered which could affect the content, and all legal disclaimers that apply to the journal pertain.

Conflict of Interest Disclosure: The authors declare that there are no conflicts of interest. 
after treatment. Ancillary biologic studies indicated Ad5/3- $\Delta 24$ replication in patients in the higher dose cohorts. All patients experienced an anti-adenoviral neutralizing antibody effect.

Conclusions-This study suggests the feasibility and safety of a serotype chimeric infectivityenhanced CRAd, Ad5/3- $\Delta 24$, as a potential therapeutic option for recurrent ovarian cancer patients.

\section{Keywords}

CRAd; gene therapy; infectivity-enhanced adenoviral vectors; ovarian cancer

\section{Introduction}

Nearly 22,000 ovarian cancer patients are diagnosed in the U.S. annually (1). With no effective screening, symptoms are often nonspecific and the majority of affected patients present with advanced disease. Though advances in surgical cytoreduction and cytotoxic chemotherapy have improved median survival, most patients develop chemotherapy resistance and ultimately succumb to their disease (2-5). Thus, there is a need for novel therapeutic approaches for this disease.

To address this need, several gene therapy strategies have been evaluated for ovarian cancer treatment (6-7). Virotherapy utilizing serotype-5 conditionally-replicative adenoviruses (CRAds) has been the basis for one such approach. This strategy takes advantage of the propensity of serotype-5 CRAds to infect cancer cells, replicate selectively within these cells, and induce cell death via oncolysis. The prototype CRAd, ONYX-015, was a serotype-5 adenovirus modified to conditionally replicate in cells with p53 deficiencies. ONYX-015 has been evaluated in early phase trials, demonstrating feasibility and safety when administered intraperitoneally (IP) to patients with recurrent ovarian cancer (8-9). However, the clinical utility of serotype-5 CRAds, such as ONYX-015, in ovarian cancer may be hampered by their inability to effectively infect ovarian cancer cells due to the paucity of the primary serotype- 5 adenoviral cell surface receptor, the coxsackie-adenovirus receptor (CAR), on their surface (10-11).

We have developed several novel approaches designed to enhance the infectivity of serotype-5 adenoviruses utilizing various CAR-independent pathways. In one of these approaches, we have modified a serotype- 5 adenovirus by replacing its knob with a serotype-3 knob onto its fiber (Supplemental Figure 1). This alters the tropism of these 5/3 serotype-chimeric adenoviruses towards serotype- 3 adenoviral receptors, which are more abundantly expressed by ovarian cancer cells (12). Our preclinical studies have demonstrated a reporter-gene-expressing Ad5/3 chimeric adenoviral vector to display higher transgene expression compared to unmodified reporter-gene-expressing adenoviral vectors in established and primary ovarian cancer cells. (13)

This 5/3 serotype-chimeric fiber knob modification has been incorporated into the CRAd, Ad5/3- $\Delta 24$ (14) (Supplemental Figure 1). Ad5/3- $\Delta 24$ contains a 24-base-pair deletion in the E1A constant region 2 (CR2) allowing for selective replication within Rb-p16-deficient tumor cells, a deficiency observed in most ovarian cancer cells (15-16). The replicative and oncolytic potency of Ad5/3- $\Delta 24$ has been confirmed in vitro in various established and primary ovarian cancer cell lines (14). Additional preclinical studies have established the antitumor activity of Ad5/3- $\Delta 24$ in in vivo ovarian cancer models (14). Biodistribution and safety studies conducted in Syrian hamsters demonstrated that IP-administered Ad5/3- $\Delta 24$ remained predominantly confined to the abdominal cavity and was well-tolerated. (17) Thus, Ad5/3-A24 seemed suited to be evaluated in early phase clinical trials in the context of gynecologic cancers. 
The primary purpose of this study was to determine the maximum tolerated dose (MTD) and toxicities associated with IP administration of Ad5/3- $\Delta 24$ in patients with selected recurrent ovarian and other select gynecologic cancers. The antitumor activity and biologic effects were also assessed.

\section{Methods}

\section{General Design}

This was a standard $3+3$ dose-escalating phase I trial evaluating IP Ad5/3- $\Delta 24$ in cohorts of eligible patients. The study was approved by both the Institutional Review Board (IRB) and the Institutional Biosafety Committee (IBC) of the University of Alabama at Birmingham (UAB). The study was also approved by the NIH Recombinant DNA Advisory Committee and the US Food and Drug Administration (FDA).

\section{Eligibility}

Eligible patients included women 19 years or older with recurrent epithelial ovarian, primary peritoneal, fallopian tube, or endometrial cancer. Patients must have been previously treated with standard surgical procedures and adjuvant therapies. Patients were required to have adequate organ function as defined by a white blood cell count $>3000 \mu \mathrm{L}$, granulocyte count $>1,500 \mu \mathrm{L}$, platelets $>100,000$, creatinine clearance $>80 \mathrm{mg} / \mathrm{dL}$, creatinine $<2.0$, aspartate aminotransferase or alanine aminotransferase $<2.5 \mathrm{x}$ the upper limit of normal reference range, bilirubin $<2.0$, and a prothrombin time/international normalized ratio or partial thromboplastin time (PT/INR/PTT) $<1.5 \mathrm{x}$ the upper limit of normal reference range. Patients were also required to have an oxygen saturation $>92 \%$ on room air, a Gynecologic Oncology Group (GOG) performance status of 0-2 and a life expectancy >3 months. Those patients found to have borderline, germ cell or sex cord/stromal tumors were excluded. Patients with recurrence solely located beyond the abdominal cavity were excluded. Patients with active heart disease (characterized by angina, unstable arrhythmia, CHF, a known EF $<5 \%$, or pulmonary hypertension), active or chronic debilitating pulmonary disease (i.e., active pneumonia, severe COPD, pulmonary edema, $\mathrm{O}_{2}$ saturation $<92 \%$ ), or coagulopathies (i.e., bleeding disorders, on therapeutic anti-coagulants) were excluded. All patients were required to sign informed consent.

Ad5/3- $\Delta 24$

Ad5/3- $\Delta 24$ is a serotype-chimeric, infectivity-enhanced adenoviral vector developed in the Gene Therapy Center at UAB (22) (Figure 1). The replication-competent Ad5- $\Delta 24$ mutant adenovirus was provided by J. Fueyo (MD Anderson Cancer Center, Houston TX). This virus contains a 24-nucleotide deletion from bp 923-946 corresponding to the amino acid sequence $\mathrm{L}_{122} \mathrm{TCHEAGF}_{129}$ of the E1A protein necessary for Rb protein binding (16). A fiber shuttle vector pNEB.PK.F5/3 containing the Ad5 tail and shaft with Ad3 knob was digested with $P a c$ I and $K p n I$, followed by cotransformation into E.coli for homologous recombination with a $S$ waI-linearized rescue plasmid containing an E1-deletion. This resulted in a plasmid with the $E 1$-deleted Ad5 genome containing the chimeric fiber domain (pTU.5/3) (18). In order to create Ad5/3- $\Delta 24$, a shuttle vector containing a 24-bp deletion in the CR2 region of $E 1 A$ (pShuttle $\Delta 24$ ) was used (19). pShuttle $\Delta 24$ was linearized with PacI/ PmeI and cotransfected into 911 cells with Pacl-linearized pTU.5/3 to rescue the Ad5/3$\Delta 24$. Propagation was performed in A549 cells. Ad5/3- $\Delta 24$ was formulated in $20 \mathrm{mM}$ Tris, $25 \mathrm{mM} \mathrm{NaCl}, 2.5 \%$ (w/v) Glycerol, $\mathrm{pH} 8.0$ (GST). Each vial contained $1 \mathrm{~mL}$ fill volume with $4.83 \times 10^{11}$ viral particles $(\mathrm{vp}) / \mathrm{mL}$ and was provided in sterile, single use containers. Ad5/3$\triangle 24$ was manufactured with support from the NCI RAID Program at the Biopharmaceutical Development Program/SAIC at NCI-Frederick. 


\section{Treatment Plan and Dose Cohorts}

Pretreatment evaluation included a history and physical examination, toxicity grading, performance status assignment, laboratory assessment (CBC with differential, chemistry profile including renal and liver function tests, PT/PTT/INR, CA-125, and anti-adenovirus neutralizing antibody titer), an abdominal-pelvic CT with tumor measurements (in two dimensions, if possible), and $\mathrm{O}_{2}$ saturation determination. Eligible patients who completed pretreatment evaluation were enrolled onto study and had an IP Quinton Curl, 22.4-inch, double-cuffed, Tenckhoff catheter (Tyco Healthcare) placed by an interventional radiologist at least one week prior to use.

Patients were assigned into one of three Ad5/3- $\Delta 24$ dose cohorts ranging from $1 \times 10^{10} \mathrm{vp} /$ dose $-1 \times 10^{12} \mathrm{vp} /$ dose (Supplemental Table 1 ). Assigned doses were administrated IP daily for three consecutive days. On days $1-4,11,18$, and 29, a history and physical, performance status, toxicity grading, CBC, and chemistry panel were obtained on all patients. CA-125 and an abdominal-pelvic CT scan were repeated on day 29.

Peritoneal aspirates, serum, saliva, and urine specimens were obtained immediately preceding Ad5/3- $\Delta 24$ administration and subsequently on days 3, 8, 15, and 29 for ancillary biologic studies. All samples were processed and de-identified prior to these studies.

\section{Evaluation of Clinical Toxicity}

Clinical toxicity was assessed on the specified days and graded with NCI Common Toxicity Criteria (CTC) Version 1.0. Dose limiting toxicity (DLT) was defined as any vector-related, grade 3, non-hematologic toxicity, or a hematologic toxicity as defined by any admission for neutropenic fever, absolute neutrophil count $<500$ for $>5$ days, or platelet count $<20,000$. The MTD was defined as the dose exceeded by the dose at which at least two patients experienced DLT.

\section{Evaluation of Clinical Efficacy}

Though not a primary objective of this study, clinical efficacy was assessed utilizing RECIST criteria, comparing pre- and post-treatment CT findings (20). Measurable disease was defined as at least one lesion $>1 \mathrm{~cm}$ that could be measured in one dimension. In each patient, up to five lesions/organ or ten lesions total were identified as target lesions. Complete response (CR) was defined as disappearance of all target lesions. Partial response (PR) was defined as $>30 \%$ decrease in the sum total recorded dimensions of a patient's target lesions. Progressive disease (PD) was deemed as $>20 \%$ increase in the sum total recorded dimensions of a patient's target lesions. Any condition that did not qualify for PR, $\mathrm{CR}$, or PD was deemed stable disease (SD). In addition, pre- and post-treatment CA-125 values were compared. Normalization of an initially elevated CA-125 was required in enrolled ovarian cancer patients if a RECIST-defined CR was noted.

\section{Assessment of Cellular Infection, Viral Replication and Generation of WildType Adenovirus in Ascites}

Total DNA from cellular material obtained from ascites samples was isolated using a QIAamp DNA MiniKit (QIAGEN) according to manufacturer protocol. Duplexing quantitative PCR was employed to evaluate Ad5/3- $\Delta 24$ infection. Ad5/3- $\Delta 24$ genome copies present in each peritoneal aspirate sample were determined by Real-Time PCR (LightCycler system, Roche Molecular Biochemicals, Indianapolis, IN) with the Ad5 hexon gene-specific forward primer 5'-TAC-GCA-CGA-CGT-GAC-CAC-A-3', reverse primer 5' ATC-CTC-ACG-GTC-CAC-AGG-G-3', and probe 6-FAM-ACC-GGT-CCC-AGC-GTTTGA-CGC-BHQ-1 and normalized to the concentration of genomic DNA by amplifying the human $\beta$-actin housekeeping gene with forward primer, reverse primer and probe: 5'-CCA- 
GCA-GAT-GTG-GAT-CAG-CA-3', 5'-CTA-GAA-GCA-TTT-GCG-GTG-GAC-3' and 6HEX-AGG-AGT-ATG-ACG-AGT-CCG-GCC-CCT-C-BHQ-1, respectively.

Ad5/3- $\Delta 24$ replication was evaluated by comparison of E1A copies as detected in the specimens of total RNA, isolated from ascites samples using the QIAamp RNeasy MiniKit (QIAGEN, Valencia, CA) and used as a template for quantitative reverse transcription PCR (RT-PCR). Duplexing RT-PCR was carried out using the TaqMan one step PCR master mix supplemented with RT enzyme (Applied Biosystems) and primers and probes specific for the E1A target gene (forward primer 5'-AAC-CAG-TTG-CCG-TGA-GAG-TTG-3', reverse primer 5'-CTC-GTT-AAG-CAA-GTC-CTC-GAT-ACA-3', probe 6-FAM-CAC-AGCCTG-GCG-ACG-CCC-A-BHQ-1) and the human $\beta$-actin housekeeping gene (above). Data was analyzed with LightCycler 480 1.5.0 SP1 software and the resultant E1A copies were normalized to the amount of human $\beta$-actin detected in the same sample to allow for comparison between patients and at different time points.

Generation of wildtype adenovirus was examined with LightCycler methodology for amplification of the $\mathrm{Ad} 5 \mathrm{E} 1$ genome without $\Delta 24$ deletion by forward and reverse primers: 5'-TGC-CAA-ACC-TTG-TAC-CGG-A-3' and 5'-CGT-CGT-CAC-TGG-GTG-GAA-A-3', and probe 6-FAM-ATC-GAT-CTT-ACC-TGC-CAC-GAG-GCT-GG-BHQ-1.

\section{Assessment for Ad5/3- $\Delta 24$ Viral Shedding}

Total DNA from Ad5/3- $\Delta 24$-treated.patients' blood, saliva, and urine specimens was isolated using the QIAamp DNA MiniKit (QIAGEN) according to manufacturer protocol. Urine specimens were initially concentrated with Millipore Amicon Ultra-4 Centrifugal Filter Units and then used to isolate viral DNA with the QIAampMinElute Virus Spin Kit (QIAGEN), according to manufacturer instructions. The DNA samples isolated from blood, saliva, and urine were used to detect the adenovirus genome by Real-Time PCR using the Ad5 hexon gene-specific forward primer 5'-TAC-GCA-CGA-CGT-GAC-CAC-A-3', reverse primer 5'-ATC-CTC-ACG-GTC-CAC-AGG G-3', and probe 6-FAM-ACC-GGTCCC-AGC-GTT-TGA-CGC-BHQ-1.

\section{Evaluation of an Anti-Adenoviral Neutralizing Antibody Response}

To evaluate for an anti-adenoviral neutralizing antibody response after treatment, a nonreplicative, luciferase-expressing virus, Ad5/3Luc1 was neutralized by either serum or ascites from Ad5/3- $\Delta 24$-treated patients before infection of SKOV3.ip1 cells. SKOV3.ip1 cells were plated into 96 -well plates (10,000 per well) and grown overnight prior to infection. A 1:50 dilution of serum or ascites from each time point specimen was prepared in $1.3 \mathrm{ml}$ of Opti-MEM media containing Ad5/3Luc1 and incubated for 30 minutes at room temperature before addition to triplicate wells at 100 plaque-forming units per cell. This infection proceeded for 48 hours and Ad5/3Luc1 transduction efficacy was determined by luciferase assay. The luciferase assay was carried out using a luciferase assay system (Promega) on an Orion microplate luminometer (Berthold) reading Culturplate-96 wells (Research Parkway) according to manufacturer protocols.

\section{Results}

\section{Patient Demographics and Treatment}

From June 2010-December 2011, 10 patients met eligibility criteria and were enrolled in the study. One patient experienced a complication related to IP catheter placement, and did not receive treatment. Table 1 summarizes patient demographics. All other nine patients completed Ad5/3- $\Delta 24$ treatment according to protocol without dose interruptions. 


\section{Toxicity Associated with Intraperitoneal Administration of Ad5/3- $\Delta 24$}

As noted, one patient had a complication related to the placement of her IP catheter and was not treated per protocol. Briefly, she developed sepsis the day after IP catheter placement from an unrecognized bowel injury, presumably encountered at the time of her IP catheter placement. In spite of catheter removal, administration of antibiotics, and aggressive resuscitation, her clinical course rapidly deteriorated and she expired 2 days after IP catheter insertion. Another patient had a grade 1 IP catheter-associated infection prior to Ad5/3- $\Delta 24$ administration that appropriately responded to antibiotics.

Table 2 summarizes all 136 reportable clinical and laboratory adverse events noted in the nine patients treated with Ad5/3- $\Delta 24$. A serious adverse event was noted in only one Ad5/3$\Delta 24$ treated patient. This patient, in the lowest dose cohort, completed Ad5/3- $\Delta 24$ therapy with acute clinical adverse events that were only grade $1 / 2$ in nature, attributed to her underlying disease. Approximately 3 weeks later, prior to her study-specified exit examination on day 29 , she developed progressive weakness and bowel dysfunction, aspirated and rapidly developed respiratory and cardiovascular compromise, requiring intensive care supportive measures. These symptoms were attributed to her underlying disease. Supportive care was eventually withdrawn after family consultation and she expired soon thereafter.

Of the 136 clinical adverse events noted, 15 were classified as attributable to Ad5/3- $\Delta 24$ treatment. These vector-attributable adverse events occurred in a total of five patients; three of the patients were in the lowest dose cohort and two were in the highest dose cohort. These events all grade 1-2 and included: fever/chills (8), nausea (3), fatigue (2), and myalgia (2). All events were transient and managed medically. There were no grade $3 / 4$ clinical DLTs attributable to $\operatorname{Ad} 5 / 3-\Delta 24$. There were no $\operatorname{Ad} 5 / 3-\Delta 24$-associated laboratory toxicities. The MTD of Ad5/3- $\Delta 24$ was not identified.

\section{Clinical Efficacy}

Antitumor activity was assessed approximately one month after IP Ad5/3- $\Delta 24$ treatment by utilizing RECIST criteria to compare pre- and post-treatment abdominal-pelvic CT examinations, and by comparing pre- and post-treatment CA-125 levels (Table 3). Of the eight Ad5/3- $\Delta 24$-treated patients evaluable for response by RECIST criteria, six (75\%) demonstrated SD while two (25\%) experienced PD. Three (37.5\%) had a decrease in their CA-125 levels and five $(62.5 \%)$ had an increase in CA-125. The three patients who had a decrease in CA-125 levels all had RECIST-defined SD. All but one of these eight patients went on to receive other therapies immediately. One patient who had stable disease and a decrease in her CA125 at the one month follow-up examination elected to not receive additional therapy; she was subsequently noted to have progressive disease 4 months after her initial treatment with Ad5/3- $\Delta 24$ and was treated with another experimental therapeutic approximately 6 months after treatment with $\operatorname{Ad} 5 / 3-\Delta 24$. The patient who experienced a serious adverse event related to respiratory/cardiac compromise was declared to have PD by virtue of clinical deterioration prior to her exit evaluation; she did not have a post-treatment CA-125.

\section{Ancillary Biologic Studies}

Various tissue samples were obtained prior to and at several time points after Ad5/3- $\Delta 24$ administration to assess for gene expression, viral shedding, generation of wildtype virus, and generation of an anti-adenovirus neutralizing antibody response. Figure 2 illustrates the detection of Ad5/3- $\Delta 24$ in ascites samples. There appeared to be a direct correlation between detectable Ad5/3- $\Delta 24$ genome copies and Ad5/3- $\Delta 24$ dose; detectable levels of Ad5/3- $\Delta 24$ vector were found in the peritoneal lavage samples obtained from most patients 
in the intermediate $\left(1 \times 10^{11} \mathrm{vp} /\right.$ dose $)$ and highest dose $\left(1 \times 10^{12} \mathrm{vp} / \mathrm{dose}\right)$ cohorts on days 3 and 8 following treatment.

To evaluate the levels of Ad5/3- $\Delta 24$ replication, total mRNA isolated from each patient's ascites sample was used to detect the early viral E1A gene message by real time PCR following reverse transcription. The detected levels of E1A mRNA (Figure 3) indicated early viral gene expression in infected cells, suggesting active Ad5/3- $\Delta 24$ replication in all patients from intermediate and highest dose cohorts on day 3 and 8 post-administration while persisting until day 15 in patients $2-1,3-1$, and 3-3 and through day 29 in patients 3-2 and 3-3. The highest levels of adenovirus replication were seen on day 3 of patients in the highest dose cohort (Figure 3), which is consistent with significantly increased number of Ad5/3- $\Delta 24$ genome copies detected in these patient's ascites (Figure 2).

Viral shedding studies (data not shown) determined that the adenoviral genomic DNA levels were significantly elevated ( $>50$ copies) in specimens from several patients including that from the blood of patient 3-3 (day 3), saliva of patient 2-1 (day 15), and urine of patients 3-1 (day 15 and 29) and 3-2 (day 3). Viral shedding was noted more frequently in patients in the intermediate and highest dose cohorts.

No emergence of wildtype adenovirus was revealed in any ascites samples (data not shown). However, a significant level of adenoviral DNA was detected in the pre-treatment ascites specimen from patient 1-1 (Figure 2).

A neutralizing anti-adenoviral antibody response was determined by blocking adenoviral gene transfer in the presence of serum or ascites samples collected from patients before and after Ad5/3- $\Delta 24$ administration. Preexisting immunity against adenovirus were noted on day 0 in the serum of patient 1-1 and the ascites of patients 1-1 through 2-3 (low and intermediate dose cohorts) (Supplemental Figure 2). A marked increase in neutralizing antibody responses, which significantly limited adenoviral gene transfer efficacy in this assay, was noted in most serum and ascites specimens collected from patients on day 8,15 , and 29 as compared to day 0 and 3 .

\section{Discussion}

Various tropism alterations to enhance the cellular infectivity of adenoviral based gene therapeutics have been accomplished. This study reports on the safety and potential efficacy of the novel infectivity-enhanced, serotype-chimeric adenovirus Ad5/3- $\Delta 24$ in patients with recurrent ovarian and select other gynecologic cancers. Incorporation of the serotype-3 knob onto the serotype-5 fiber in this CRAd enhances infectivity and improves oncolysis in ovarian cancer models (12-14). In this study, we were able to safely administer Ad5/3- $\Delta 24$ IP daily for three consecutive days at dosages up to $1 \times 10^{12} \mathrm{vp} / \mathrm{dose}$.

The clinical toxicities attributable to Ad5/3- $\Delta 24$ were manageable grade $1-2$ constitutional or pain symptoms. Attributable toxicities were scattered between the lowest and highest cohorts and there did not appear to be a relationship between adverse events and Ad5/3- $\Delta 24$ dose. No Ad5/3- $\Delta 24$-specific laboratory abnormalities were noted; there were no grade $3 / 4$ clinical or laboratory DLT directly attributable to Ad5/3- $\Delta 24$. Manufacturing constraints did not allow for Ad5/3- $\Delta 24$ cohorts at higher dosages; thus, the MTD of Ad5/3- $\Delta 24$ was not identified in this trial.

Most other adverse events experienced by Ad5/3- $\Delta 24$-treated patients were attributable to their underlying disease. Of note, one patient enrolled on the study experienced a serious bowel perforation related to IP catheter placement and did not receive treatment. Only one other patient among over 60 patients treated in a series of four other phase I gene therapy 
trials of ours has experienced a bowel perforation (21-24). In that instance, the bowel injury was immediately recognized and the IP catheter immediately removed. She recovered uneventfully with the administration of intravenous antibiotics.

Though this trial was not primarily designed to determine clinical efficacy, six patients (75\%) had RECIST-defined SD one month after treatment and three patients $(37.5 \%)$ had a decrease in CA-125. All three patients who had a decrease in CA-125 had RECIST-defined SD one month after treatment. All but one treated study patient went on to receive different treatment for their disease right after the one-month follow-up evaluation. The one patient who was observed was noted to have progressive disease 4 months after Ad5/3- $\Delta 24$ treatment. The true clinical efficacy of $A d 5 / 3-\Delta 24$ will need to be assessed in phase II studies evaluating expanded cycles of Ad5/3- $\Delta 24$.

These results mimic those of other trials evaluating novel infectivity-enhanced CRAds in the context of ovarian cancer. Koski et al. reported their phase I study evaluating the safety and potential efficacy of a similar serotype-chimeric infectivity-enhanced CRAd (25). In this trial, 21 patients with a variety of solid tumors were treated with a single intratumoral or intravenous administration of up to $4 \times 10^{11}$ vp of an Ad5/3- $\Delta 24$-modified CRAd expressing GMCSF. Low-dose cyclophosphamide was also administered to reduce regulatory T-cells. Grade 1-2 flu-like symptoms, injection site discomfort and abdominal pain were the most common clinical effects. Four patients with ovarian cancer were included in this trial; one was noted to have RECIST-defined SD and one other patient had a minor CA-125 response.

Our prior phase I trial investigated the safety, potential antitumor effect, and biologic activity of the infectivity-enhanced CRAd, Ad5- $\Delta 24-$ RGD, in patients with recurrent selected gynecologic cancers (23). This Ad5- $\Delta 24$-based CRAd incorporated an Arg-GlyAsp (RGD) sequence in the HI loop of the serotype-5 fiber knob, redirecting viral tropism towards $\alpha_{\nu} \beta$-integrins, abundantly expressed on ovarian cancer cell surfaces $(10,11)$. Preclinical studies demonstrated that this RGD modification enhanced the viral infectivity and antitumor activity of Ad5- $\Delta 24-$ RGD in various ovarian cancer models (26-27). In a phase I trial, 21 patients with recurrent selected gynecologic cancers were treated IP with Ad5- $\Delta 24-$ RGD at dosages of up to $1 \times 10^{12} \mathrm{vp} / \mathrm{d}$ daily for three days (23). Toxicities were limited to grades $1 / 2$ fever, fatigue or pain. Fifteen patients ( $71 \%$ ) were noted to have SD and seven patients (33\%) were noted to have a decrease in CA-125. Similar clinical efficacy and toxicity were noted in a separate phase I trial investigating the RGD-modified nonreplicative suicide gene-based serotype-5 adenovirus, Ad5.SSTR/TK.RGD (24).

Ancillary studies demonstrated a consistent dose-related increase in detectable Ad5/3- $\Delta 24$ vector and its replication in ascites samples with the highest concentrations being noted within the highest dose cohort. The detection and replication of adenovirus in ovarian cancer patients treated with a GMCSF-expressing Ad5/3- $\Delta 24$ was not detailed in the early phase trial by Koski et al (25). The results of the current study were comparable to our prior early phase trial evaluating Ad5- $\Delta 24-$ RGD. In that trial, RGD-specific PCR demonstrated the presence of study vector in the ascetic fluid of 16 of 21 treated patients (23).

Viral shedding studies in the current investigation detected elevated Ad genomic DNA levels (>50 copies) in serum, saliva, and urine from only a few patients, mostly from the higher dose cohorts. Wildtype adenovirus emergence after Ad5/3- $\Delta 24$ treatment was not noted. A robust anti-viral antibody response was also noted. These findings were comparable to that reported by Koski et al and in our prior early phase trials evaluating RGD-modified infectivity-enhanced gene therapeutics (23-25). Preexisting neutralizing anti-adenoviral antibody levels were noted on day 0 in the serum of patients from the low and intermediate dose cohorts. Several studies have indicated that neutralizing antibodies, in 
addition to other specific and non-specific factors, can interact with adenoviral vectors and may impair gene transfer and virus amplification in the tumor milieu (28-30). The question remains which of these factors present in malignant ascites can affect Ad5/3 vector infectivity in specific or nonspecific manners and, thus, limit the efficacy of oncolytic Ad5/3- $\Delta 24$ in patients. Answering the above question requires detailed analysis of infectivity of Ad5/3 vectors in the context of whole ascites, which was beyond the scope of this trial.

In conclusion, this study demonstrates that a serotype-chimeric infectivity-enhanced CRAd such as Ad5/3- $\Delta 24$ can be safely administered IP to patients with recurrent ovarian and other selected gynecologic cancers daily for three consecutive days at dosages up to $1 \times 10^{12}$ vp. Vector-related toxicities at the doses evaluated were mild, manageable, and transient. While this was not a phase II trial designed to evaluate efficacy, SD and declines in CA-125 were noted in several patients. Evidence of gene transfer, adenoviral replication, and a robust anti-adenoviral antibody response were noted. Efforts are underway to incorporate novel non-invasive imaging capacity into Ad5/3- $\Delta 24$. Other preclinical studies have demonstrated the potential synergy of $A d 5 / 3-\Delta 24$ in combination with conventional chemotherapy and randomized phase II trials of chemotherapy with or without Ad5/3- $\Delta 24$ might also be justified (31). Further development and investigation of these novel CRAd approaches in the context of ovarian cancer appears to be warranted.

\section{Supplementary Material}

Refer to Web version on PubMed Central for supplementary material.

\section{Acknowledgments}

Financial Support: This project has been funded in whole or in part with funds from the National Cancer Institute, National Institutes of Health, under Contract No. HHSN261200800001E. The content of this publication does not necessarily reflect the views of policies of the Department of Health and Human Services, nor does mention of trade names, commercial products, or organizations imply endorsement by the U.S. Government. This research was also supported by the UAB Comprehensive Cancer Center's Clinical Protocol and Data Management Shared Facility (P30 CA013148).

\section{References}

1. Siegel R, Naishadham D, Jemal A. Cancer statistics, 2012. CA Cancer J Clin. 2012; 62:10-29. [PubMed: 22237781]

2. McGuire WP, Hoskins WJ, Brady MF, Kucera PR, Partridge EE, Look KY, et al. Cyclophosphamide and cisplatin compared with paclitaxel and cisplatin in patients with stage III and stage IV ovarian cancer. N Engl J Med. 1996; 334:1-6. [PubMed: 7494563]

3. Ozols RF, Bundy BN, Greer BE, Fowler JM, Clarke-Pearson D, Burger RA, Gynecologic Oncology Group, et al. Phase III trial of carboplatin and paclitaxel compared with cisplatin and paclitaxel in patients with optimally resected stage III ovarian cancer: a Gynecologic Oncology Group study. J Clin Oncol. 2003; 21:3194-3200. [PubMed: 12860964]

4. Bristow RE, Berek JS. Surgery for ovarian cancer: how to improve survival. Lancet. 2006; 367:1558-1560. [PubMed: 16698396]

5. Armstrong DK, Bundy B, Wenzel L, Huang HQ, Baergen R, Lele S, et al. Intraperitoneal cisplatin and paclitaxel in ovarian cancer. N Engl J Med. 2006; 354:34-43. [PubMed: 16394300]

6. Kimball KJ, Numnum TM, Rocconi RP, Alvarez RD. Gene therapy for ovarian cancer. Curr Oncol Rep. 2006; 8:441-447. [PubMed: 17040622]

7. Kanerva A, Raki M, Hemminki A. Gene therapy of gynaecological diseases. Expert Opin Biol Ther. 2007; 7:1347-1361. [PubMed: 17727325] 
8. Nemunaitis J, Cunningham C, Buchanan A, Blackburn A, Edelman G, Maples P, et al. Intravenous infusion of a replication-selective adenovirus (ONYX-015) in cancer patients: safety, feasibility, and biological activity. Gene Therapy. 2001; 8:746-759. [PubMed: 11420638]

9. Vasey PA, Schulman LN, Campos S, Davis J, Gore M, Johnston S, et al. Phase I trial of intraperitoneal injection of the E1B-55kd-gene deleted adenovirus ONYX-015 (d11520) given on days 1 through 5 every 3 weeks in patients with recurrent/refractory epithelial ovarian cancer. J Clin Oncol. 2002; 20:1562-1569. [PubMed: 11896105]

10. Douglas JT, Kim M, Sumerel LA, Carey DE, Curiel DT. Efficient oncolysis by a replicating adenovirus (ad) in vivo is critically dependent on tumor expression of primary Ad receptors. Ca Res. 2001; 61:813-817.

11. Kim M, Zinn KR, Barnett BG, Sumerel LA, Krasnykh V, Curiel DT, et al. The therapeutic efficacy of adenoviral vectors for cancer gene therapy is limited by a low level of primary adenovirus receptors on tumour cells. Eur J Cancer. 2002; 38(14):1917-1926. [PubMed: 12204675]

12. Kanerva A, Mikheeva GV, Krasnykh V, Coolidge CJ, Lam JT, Mahasreshti PJ, et al. Targeting adenovirus to the serotype 3 receptor increases gene transfer efficiency to ovarian cancer cells. Clin Cancer Res. 2002; 8(1):275-280. [PubMed: 11801569]

13. Kanerva A, Wang M, Bauerschmitz GJ, Lam JT, Desmond RA, Bhoola SM, et al. Gene transfer to ovarian cancer versus normal tissues with fiber-modified adenoviruses. Mol Ther. 2002; 5(6):695704. [PubMed: 12027553]

14. Kanerva A, Zinn KR, Chaudhuri TR, Lam JT, Suzuki K, Uil TG, et al. Enhanced therapeutic efficacy for ovarian cancer with a serotype 3 receptor-targeted oncolytic adenovirus. Mol Ther. 2003; 8(3):449-458. [PubMed: 12946318]

15. Heise C, Hermiston T, Johnson L, Brooks G, Sampson-Johannes A, Williams A, et al. An adenovirus E1A mutant that demonstrates potent and selective systemic anti-tumoral efficacy. Nat Med. 2000; 6(10):1134-1139. [PubMed: 11017145]

16. Fueyo J, Gomez-Manzano C, Alemany R, Lee PS, McDonnell TJ, Mitlianga P, et al. A mutant oncolytic adenovirus targeting the $\mathrm{Rb}$ pathway produces anti-glioma effect in vivo. Oncogene. 2000; 19(1):2-12. [PubMed: 10644974]

17. Kim KH, Ryan MJ, Estep JE, Miniard BM, Rudge TL, Peggins JO, et al. A new generation of serotype chimeric infectivity-enhanced conditionally replicative adenovirals: the safety profile of Ad5/3- $\Delta 24$ in advance of a phase I clinical trial in ovarian cancer patients. Hum Gene Ther. 2011; 22(7):821-828. [PubMed: 21171861]

18. Uil TG, Seki T, Dmitriev I, Kashentseva E, Douglas JT, Rots MG, et al. Generation of an adenoviral vector containing an addition of a heterologous ligand to the serotype 3 fiber knob. Cancer Gene Ther. 2003; 10(2):121-124. [PubMed: 12536200]

19. Suzuki K, Alemany R, Yamamoto M, Curiel DT. The presence of the adenovirus E3 region improves the oncolytic potency of conditionally replicative adenoviruses. Clin Cancer Res. 2002; 8(11):3348-3359. [PubMed: 12429621]

20. Rustin GJ, Vergote I, Eisenhauer E, Pujade-Lauraine E, Quinn M, Thigpen T, et al. Gynecological Cancer Intergroup Definitions for response and progression in ovarian cancer clinical trials incorporating RECIST 1.1 and CA 125 agreed by the Gynecological Cancer Intergroup (GCIG). Int J Gynecol Cancer. 2011; 21:419-423. [PubMed: 21270624]

21. Alvarez RD, Curiel DT. A phase I study of recombinant adenovirus vector-mediated intraperitoneal delivery of herpes simplex virus thymidine kinase (HSV-TK) gene and intravenous ganciclovir for previously treated ovarian and extraovarian cancer patients. Hum Gene Ther. 1997; 8(5):597-613. [PubMed: 9095411]

22. Alvarez RD, Barnes MN, Gomez-Navarro J, Wang M, Strong TV, Arafat W, et al. A cancer gene therapy approach utilizing an anti-erbB-2 single-chain antibody-encoding adenovirus (AD21): a phase I trial. Clin Cancer Res. 2000; 6(8):3081-3087. [PubMed: 10955787]

23. Kimball KJ, Preuss MA, Barnes MN, Wang M, Siegal GP, Wan W, et al. A phase I study of a tropism-modified conditionally replicative adenovirus for recurrent malignant gynecologic diseases. Clin Cancer Res. 2010; 16(21):5277-5287. [PubMed: 20978148] 
24. Kim KH, Dmitriev I, O'Malley JP, Wang M, Saddekni S, You Z, et al. A phase I clinical trial of Ad5.SSTR/TK.RGD, a novel infectivity-enhanced bicistronic adenovirus, in patients with recurrent gynecologic cancer. Clin Cancer Res. 2012; 18(12):3440-3451. [PubMed: 22510347]

25. Koski A, Kangasniemi L, Escutenaire S, Pesonen S, Cerullo V, Diaconu I, et al. Treatment of cancer patients with a serotype 5/3 chimeric oncolytic adenovirus expressing GMCSF. Mol Ther. 2010; 18(10):1874-1884. [PubMed: 20664527]

26. Bauerschmitz G, Lam J, Kanerva A, Suzuki K, Nettelbeck DM, Dmitriev I, et al. Treatment of ovarian cancer with a tropism modified oncolytic adenovirus. Cancer Res. 2002; 62:1266-1269. [PubMed: 11888888]

27. Page JG, Tian B, Schweikart K, Tomaszewski J, Harris R, Broadt T, et al. Identifying the safety profile of a novel infectivity-enhanced conditionally replicative adenovirus, Ad5-delta24-RGD, in anticipation of a phase I trial for recurrent ovarian cancer. Am J Obstet Gynecol. 2007; 196:389e110. [PubMed: 17403430]

28. Bernal RM, Sharma S, Gardner BK, Douglas JT, Bergelson JM, Dubinett SM, et al. Soluble coxsackie adenovirus receptor is a putative inhibitor of adenoviral gene transfer in the tumor milieu. Clin Cancer Res. Jun; 2002 8(6):1915-1923. [PubMed: 12060636]

29. Hara T, Kuriyama S, Kiyohara H, Nagase Y, Matsumoto M, Seya T. Soluble forms of membrane cofactor protein (CD46, MCP) are present in plasma, tears, and seminal fluid in normal subjects. Clin Exp Immunol. Sep; 1992 89(3):490-494. [PubMed: 1516264]

30. Wang H, Beyer I, Persson J, Song H, Li Z, Richter M, et al. A new human DSG2-transgenic mouse model for studying the tropism and pathology of human adenoviruses. J Virol. Jun; 2012 86(11): 6286-6302. [PubMed: 22457526]

31. Raki M, Sarkioja M, Desmond RA, Chen DT, Butzow R, Hemminki A, et al. Oncolytic adenovirus Ad5/3-delta24 nd chemotherapy for treatment of orthotopic ovarian cancer. Gynecol Oncol. 2008; 108(1):166-172. [PubMed: 17950450] 


\section{Research Highlights}

- $\quad \operatorname{Ad} 5 / 3-\Delta 24$ is a serotype chimeric, infectivity enhanced CRAd

- $\quad \operatorname{Ad} 5 / 3-\Delta 24$ has shown oncolysis and antitumor activity in preclinical ovarian cancer models

- $\quad$ Recurrent ovarian \& other select gynecologic cancer patients were treated with IP Ad5/3- $\Delta 24$

- $\quad$ At the doses evaluated, $\operatorname{Ad} 5 / 3-\Delta 24$ is a safe potential therapeutic option in these patients 


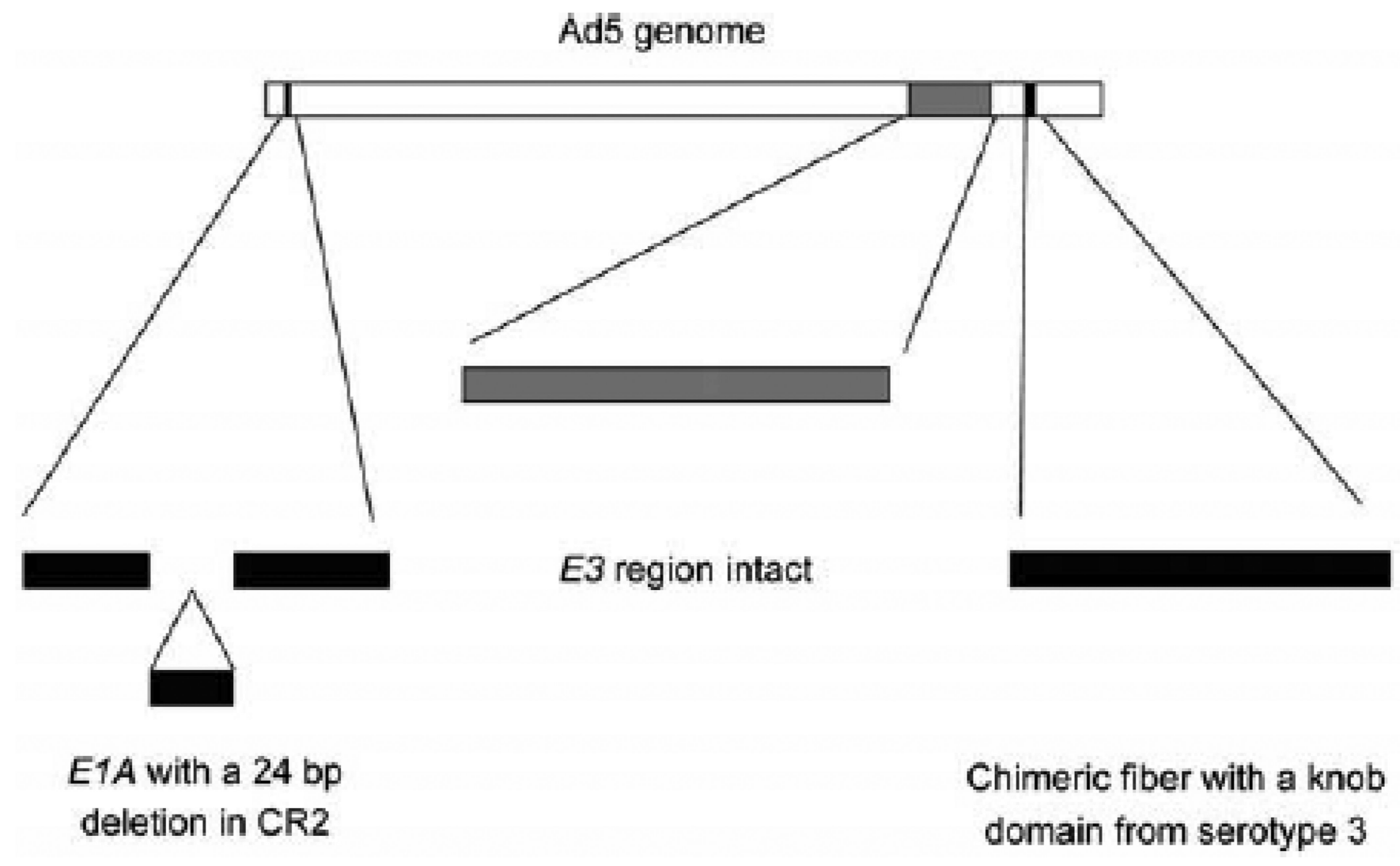

Figure 1.

Structure of Ad5/3- $\Delta 24$. (A) Ad5/3- $\Delta 24$ has a 24 bp deletion in the constant region 2 (CR2) of the E1 A gene, corresponding to the region utilized for $\mathrm{Rb}$ protein binding. This results in an agent that is replication-competent and oncolytic in cells defective in the $\mathrm{Rb} / \mathrm{p} 16$ pathway, such as ovarian cancer cells. The fiber is modified to incorporate the serotype 3 knob, while retaining the Ad5 shaft and tail. 


\section{Ad presence in patient ascites}

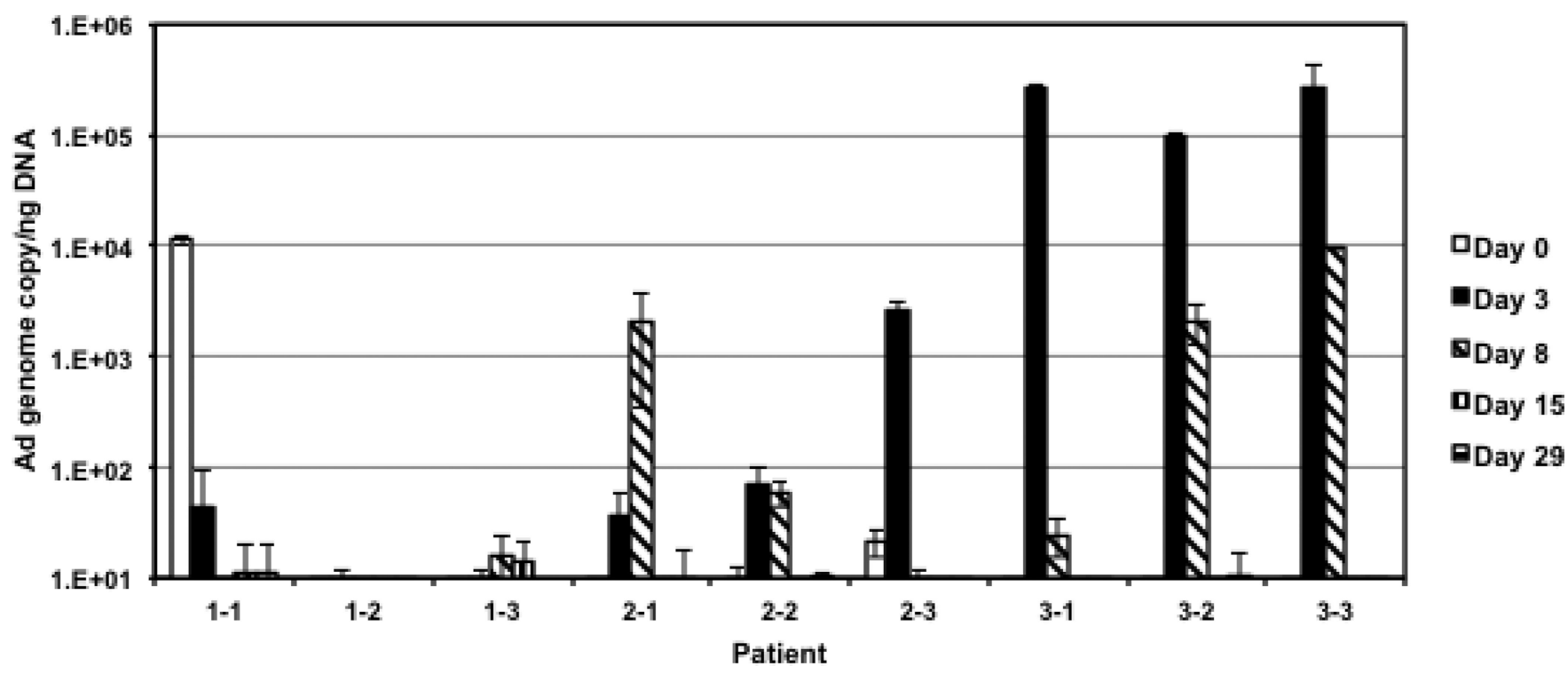

Figure 2.

Quantification of Ad5/3- $\Delta 24$ vector in ascites samples. The Ad5/3- $\Delta 24$ genome copies were quantified (in triplicate) in each patient's ascites specimen and normalized to the amount of cellular DNA detected in the same sample with primers and probe for human $\beta$-actin using duplexing quantitative PCR settings. 


\section{Ad replication in patient ascites}

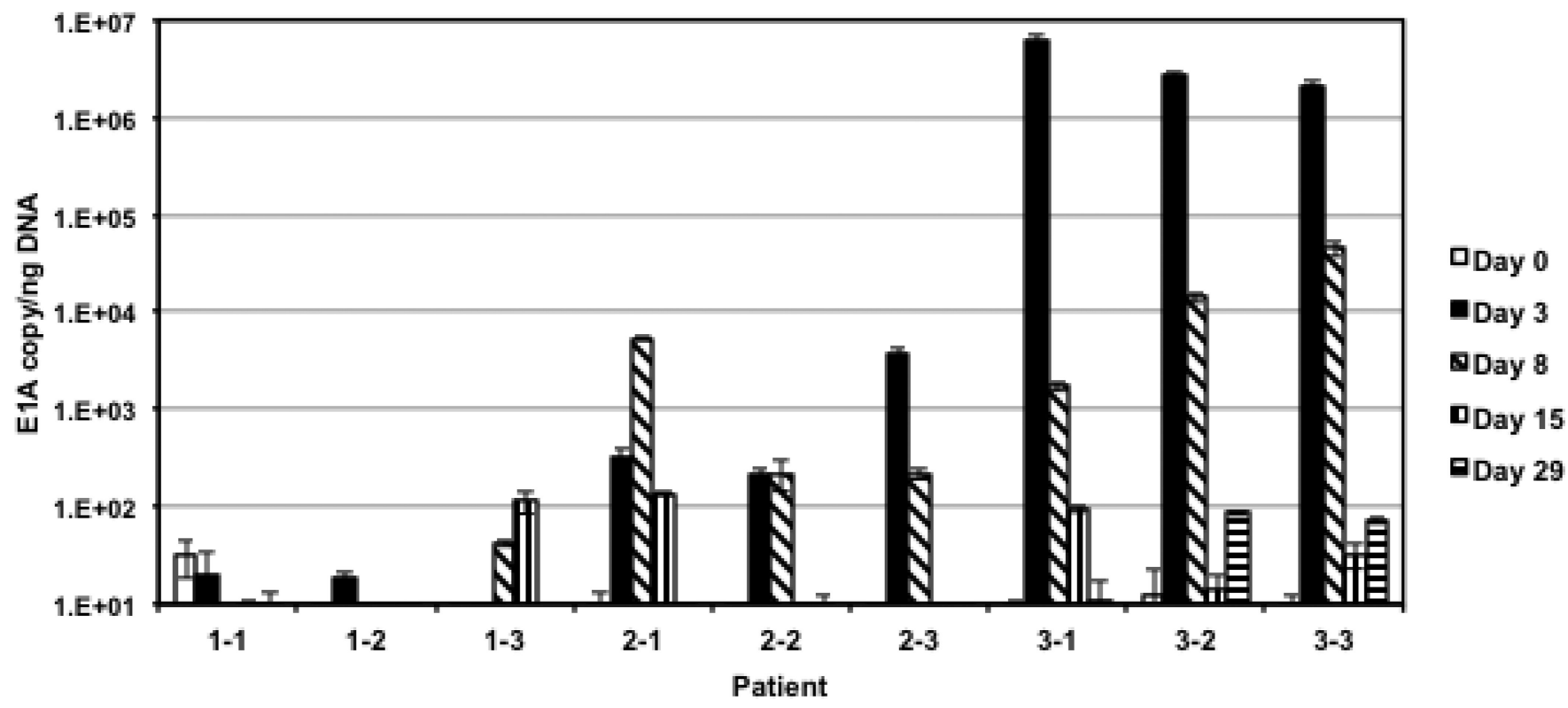

Figure 3.

Assessment of Ad5/3- $\Delta 24$ replication in ascites. The early E1A gene-specific mRNA was quantified (in triplicate) using total mRNA isolated from each patient's ascites specimen as a template for reverse transcription followed by quantitative PCR. The determined copy number of E1A mRNA was normalized to the amount of human $\beta$-actin, which was detected in the same sample using duplexing RT-PCR settings. 
Table 1

Patient characteristics $(n=9)$.

\begin{tabular}{|l|c|}
\hline Age (years) & 63.5 \\
Median & $53-84$ \\
Range & $\mathrm{N}(\%)$ \\
\hline Race & $10(100)$ \\
Caucasian & 0 \\
African American & 0 \\
Other & $\mathrm{N}(\%)$ \\
\hline Primary cancer & $7(78)$ \\
Ovarian & $1(11)$ \\
Fallopian tube & $1(11)$ \\
Primary peritoneal & $\mathrm{N}$ \\
\hline Prior chemotherapy regimens & 4 \\
Median & $1-10$ \\
Range & \\
\hline
\end{tabular}




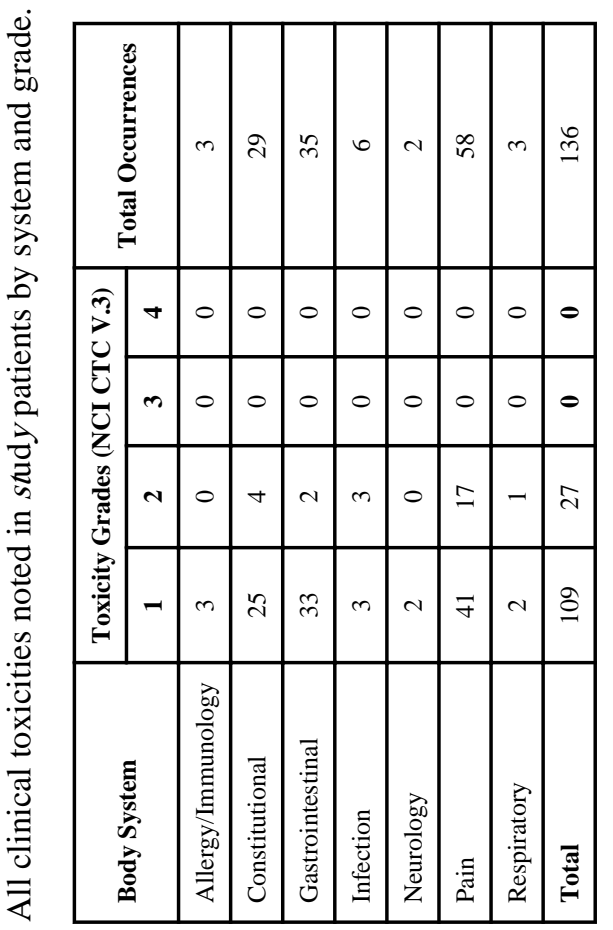


Table 3

Clinical efficacy by RECIST criteria and pre- and post-treatment CA125 levels.

\begin{tabular}{|c|c|c|c|}
\hline Patient & $\begin{array}{c}\text { RECIST-defined } \\
\text { disease response }\end{array}$ & Pre-treatment CA-125 & Post-treatment CA-125 \\
\hline 101 & Stable & 65.9 & 44.2 \\
\hline 102 & Progressive & 5052.7 & 52,520 \\
\hline $103^{*}$ & Not determined & 1465.0 & Not determined \\
\hline $201^{* *}$ & Not applicable & 60.7 & Not applicable \\
\hline 202 & Stable & 62.7 & 20.6 \\
\hline 203 & Progressive & 126.2 & 134.6 \\
\hline 204 & Stable & 130.5 & 315.7 \\
\hline 301 & Stable & 284.0 & 246.4 \\
\hline 302 & Stable & 189.4 & 196.3 \\
\hline 303 & Stable & 127.4 & 229.5 \\
\hline
\end{tabular}

* Noted as progression based on clinical deterioration

*** Did not receive study treatment due to IP catheter complication 